\title{
Das Gefühl des Beschmutztseins bei erwachsenen Opfern sexualisierter Gewalt in Kindheit und Jugend
}

\author{
Kerstin Jung $^{\mathrm{a}} \quad$ Anne Dyer $^{\mathrm{b}} \quad$ Kathlen Priebe $^{\mathrm{c}} \quad$ Ulrich Stangier $^{\mathrm{a}} \quad$ Regina Steil $^{\mathrm{a}}$

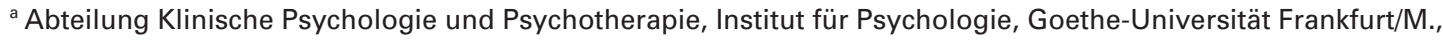

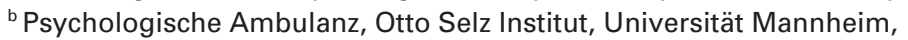 \\ ${ }^{c}$ Klinik für Psychosomatische Medizin und Psychotherapie am Zentralinstitut für Seelische Gesundheit Mannheim, Deutschland
}

\section{Schlüsselwörter}

Posttraumatische Belastungsstörung · Sexueller Missbrauch · Imagination · Gefühl des Beschmutztseins . Ekel · Mental Pollution · Kurzintervention

\section{Zusammenfassung}

Das Gefühl des Beschmutztseins stellt ein weit verbreitetes Phänomen infolge sexualisierter Gewalt dar, das bislang in der Forschung vernachlässigt wurde. Ziel des Artikels ist es, dieses Symptom nach sexuellem Missbrauch zu beschreiben und die Notwendigkeit seiner gezielten Behandlung abzuleiten. Dabei wird auf die Phänomenologie, Konsequenzen und mögliche Erklärungsansätze eingegangen. Ein neu entwickeltes, ökonomisches Interventionsprogramm zur spezifischen Behandlung dieses Erlebens bei erwachsenen Opfern sexualisierter Gewalt in Kindheit und Jugend wird vorgestellt. Eine Falldarstellung veranschaulicht die Wirkung der kurzen Intervention, die sich in eine Gesamtbehandlung gut integrieren lässt. In der Falldarstellung resultiert die Behandlung des Gefühls des Beschmutztseins darüber hinaus in einer Reduktion der Symptomatik der Posttraumatischen Belastungsstörung.

\section{Phänomenologie}

Klinische Erfahrung und erste Studien [z.B. Fairbrother und Rachman, 2004] zeigen, dass Opfer sexualisierter Gewalt neben den klassischen Symptomen der Posttraumatischen Belastungsstörung (PTBS) häufig unter einem starken Gefühl der Beschmutztheit leiden. So beschrieben in einer Studie von

\section{Keywords}

Posttraumatic stress disorder . Sexual abuse .

Imagery - Feeling of being contaminated - Disgust .

Mental pollution . One-session program

\section{Summary}

The Feeling of Being Contaminated in Adult Survivors of Childhood Sexual Abuse

Many survivors of childhood sexual abuse (CSA) suffer from a distressing feeling of being contaminated. So far, this symptom is being neglected in research. The aim of this article is to illustrate this symptom and the necessity of a specialized treatment. Phenomenology, consequences, and possible concepts of explanation are described. The paper presents a newly developed shorttime treatment of the feeling of being contaminated in adult survivors of CSA. A case study demonstrates the outcome of the one-session program which can easily be integrated in a whole treatment program. The case study shows that the treatment results furthermore in a reduction of the symptoms of posttraumatic stress disorder.

\begin{tabular}{ll}
\hline KARGER & @ 2011 S. Karger GmbH, Freiburg \\
Fax +497614520714 & 1016-6262/11/0214-0247\$38.00/0 \\
Information@Karger.de & $\begin{array}{l}\text { Accessible online at: } \\
\text { www.karger.com }\end{array}$ \\
www.karger.com/ver
\end{tabular}

Fairbrother und Rachman [2004] mehr als 60\% der Frauen, die sexuellen Missbrauch im Erwachsenenalter erlebt hatten (14\% mit resultierender PTBS), ein Gefühl der Beschmutztheit, das nach dem traumatischen Ereignis zu gesteigertem Waschverhalten führte. Es ist davon auszugehen, dass dieses Symptom insbesondere im Rahmen einer komplexen PTBSSymptomatik auftritt. So listet Herman [1992] in ihrem Vor- 
schlag der Diagnose einer komplexen PTBS dezidiert die dysfunktionale Überzeugung von Missbrauchsopfern beschmutzt zu sein als ein mögliches Symptom auf. Klinische Erfahrung zeigt jedoch, dass durchaus auch Opfer einmaliger sexueller Übergriffe und Opfer sexualisierter Gewalt im Erwachsenenalter ein Gefühl des Beschmutztseins entwickeln können.

Bisherige Forschung zum Gefühl der Beschmutztheit orientiert sich an einem von Rachman [1994] entwickelten Konstrukt der «Mental Pollution». Dieses ist definiert durch 6 Merkmale [Fairbrother und Rachman, 2004]: 1) ein Gefühl der inneren Beschmutztheit, 2) Aktivierung ohne wirklichen Kontakt mit realem Schmutz, 3) Andauern auch ohne Kontakt mit realem Schmutz, 4) unzureichende Reduktion durch Waschen, 5) Aktivierung durch Gedanken, Bilder, Worte oder Erinnerungen und 6) einen moralischen Aspekt (z.B. begleitende Gefühle von Schuld und Scham). In einer empirischen Studie von Fairbrother und Rachman [2004] trafen durchschnittlich jedoch nur 3 der postulierten 6 Kriterien des Gefühls beschmutzt zu sein zu. Weitere Studien zur phänomenologischen Exploration dieses Phänomens sind indiziert. Betroffene berichten von der Überzeugung, noch Jahrzehnte nach der Erfahrung sexualisierter Gewalt durch das Erlebte befleckt zu sein. Dies geht unserer klinischen Erfahrung nach mit dem Eindruck einher, dass der Geruch von Sperma nach wie vor auf der Haut hafte, oder mit der Wahrnehmung eines schmutzigen Films, der den gesamten Körper bedeckt und nicht entfernt werden kann [Fairbrother, 2002]. Einige Betroffene klagen Dekaden nach den Übergriffen über ein klebriges Empfinden insbesondere im Genital-, Brust- und Mundbereich. Andere geben an, dass das Gefühl des Beschmutztseins in starkem Maß auf einer Überzeugung basiere und keine sensorischen Anteile aufweise. In der Regel berichten die Betroffenen, dass ein Kennzeichen des Gefühls des Beschmutztseins darin bestehe, dass es immer vorhanden sei, durch Auslöser jedoch zunehmen oder stärker bewusst werden könne. Typische Auslöser seien Erinnerungen an das Trauma [Fairbrother und Rachman, 2004], soziale Kontakte, interpersonelle Nähe, sexuelle Intimität, Leistungssituationen, aber auch die Wahrnehmung des eigenen Körpergeruchs.

\section{Konsequenzen und dysfunktionales Copingverhalten}

Das Gefühl des Beschmutztseins nach sexualisierter Gewalt geht mit unterschiedlichen Emotionen (Scham, Selbstverachtung, Schuld und anderen) einher [Fairbrother, 2002]. Eine weit verbreitete Konsequenz ist ausgeprägter Ekel vor dem eigenen Körper. Insbesondere die eigenen Brüste und der Genitalbereich - bei stattgefundenem oralem Missbrauch auch der Mund - werden hierbei als aversiv erlebt. Betroffene berichten von Schwierigkeiten, die als schmutzig erlebten Körperpartien beim Duschen anzufassen. Sehr viel häufiger scheinen Missbrauchsopfer auf das Gefühl des Beschmutzt- seins aber mit ausgeprägtem, zwanghaft anmutendem Waschverhalten zu reagieren [Fairbrother, 2002]. Dabei können die Reinigungsversuche bis zur Bearbeitung der Haut mit ätzenden Substanzen wie Essig und Desinfektionsmitteln und Schrubben der Haut mit Bürsten oder der Verwendung stark parfümierter Pflegeprodukte [Fairbrother, 2002] reichen.

Vor dem Hintergrund einer Traumatisierung erhöht sich die Wahrscheinlichkeit, eine Zwangsstörung zu entwickeln [DeSilva und Marks, 1999]. Andererseits zeigt die klinische Erfahrung, dass das Waschverhalten vieler Missbrauchsopfer zwar zwanghaft anmutet, aber eindeutig auf das Gefühl des Beschmutztseins nach Traumatisierung zurückgeht. Bei ausbleibender Generalisierung (z.B. Waschen aufgrund einer befürchteten, nicht vom eigenen Körper ausgehenden Kontamination, Entwicklung von Kontrollzwängen) sollte das auffällige Waschverhalten folglich nicht als Zwang, sondern als eine Meidungs- oder Copingstrategie im Rahmen der PTBS beschrieben werden.

Da in der klinischen Praxis zahlreiche Betroffene angeben, dass «beschmutzt sein» mit «schlecht sein» oder «wertlos sein» gleichzusetzen sei [Rachman, 2006], stellen Scham und Selbstverachtung naheliegende resultierende Emotionen dar [Fairbrother, 2002]. Neben dem exzessiven Reinigungsverhalten reagieren Betroffene mit Ablenkungsstrategien. Tritt es in sozialen Situationen auf, werden diese verlassen. Der Meidung von und Flucht aus sozialen Situationen liegt bei einigen Patienten die Überzeugung zugrunde, andere Menschen ihres Umfeldes könnten die Beschmutztheit bemerken und sich in der Folge vor ihnen ekeln und abwenden [Rachman, 2004]. Weiterhin vermeiden Opfer des Gefühls des Beschmutztseins häufig die Auseinandersetzung mit dem eigenen Körper und sind in ihrem sexuellen Erleben und Verhalten beeinträchtigt.

\section{Fehlen eines Behandlungsansatzes und theoretische Hintergründe zur Pathogenese}

Trotz des dargestellten Leidensdrucks und den häufig resultierenden dysfunktionalen Bewältigungsstrategien existieren bislang keine Behandlungsansätze, die sich speziell dem Gefühl des Beschmutztseins widmen. Auf die Notwendigkeit der Entwicklung einer gezielten Intervention über die etablierte Behandlung der PTBS hinaus weist jedoch eine Falldarstellung von Fairbrother und Rachman [2004] hin: In dieser bleibt nach erfolgreicher Behandlung der PTBS-Symptomatik das Gefühl der Beschmutztheit weiterhin bestehen [DeSilva und Marks, 1999]. Darüber hinaus zeigen Gershuny et al. [2002], dass bei Patienten mit Waschzwängen die klassische Intervention der Exposition und Reaktionsverhinderung bei gleichzeitigem Bestehen einer PTBS erfolglos bleibt. Hier könnte das zwanghaft anmutende Waschverhalten einiger Patienten auf das Bestehen eines nicht behandelten Gefühls des Beschmutztseins nach sexualisierter Gewalt zurückzuführen 
sein. Darüber hinaus fordert Rachman [2004] für Kontaminationsängste, dass die State-of-the-Art-Expositionsverfahren aufgrund eher ungünstiger Remissions- und hoher Rückfallraten grundsätzlich um weitere Elemente ergänzt werden sollten. Explizit benennt er hierbei den potenziellen Nutzen kognitiver Verfahren.

Das Gefühl der Beschmutztheit oder Kontamination wird gemäß Rachman [2004] als ein interpretativer Prozess verstanden, der dem Erleben von Ekel und den assoziierten Emotionen Scham und Selbstverachtung [Power und Dalgleish, 2008] zugrunde liegt. Welche Wirkmechanismen an der Entstehung und Aufrechterhaltung des Gefühls beschmutzt zu sein beteiligt sind, ist bislang ungeklärt. Einerseits könnte davon ausgegangen werden, dass Ekel und Abneigung durch Klassische Konditionierung an diverse zuvor neutrale Reize (wie den eigenen Körper) gekoppelt werden. Dies wäre dann naheliegend, wenn Betroffene angeben, dass sie sich bereits während der Traumatisierung beschmutzt gefühlt haben. Im Rahmen der Hypothese zur Klassischen Konditionierung können Schilderungen zu wahrgenommenen Gerüchen und Körpersensationen als sensorische Intrusionen im Rahmen der PTBS-Symptomatik gewertet werden. Für die Hypothese der Klassischen Konditionierung sprechen Studien, die zeigen, dass Ekel ein weit verbreitetes Gefühl während unterschiedlicher traumatischer Erlebnisse $\mathrm{zu}$ sein scheint und während des Erlebens sexualisierter Gewalt in Kindheit und Jugend in stärkerem Ausmaß auftritt als während des Erlebens physischer Gewalt [Hathaway et al., 2010]. Ein zweiter, alternativer Erklärungsansatz geht davon aus, dass das Gefühl beschmutzt zu sein auf einer massiv negativen Interpretation der Konsequenzen des Traumas basiert (z.B. für immer beschmutzt, eklig, wertlos, verdorben sein) [Ehlers und Clark, 2000]. Für den zweiten Wirkmechanismus spricht, wenn Betroffene angeben, dass das Gefühl der Beschmutztheit erst Jahre nach dem sexuellen Missbrauch erstmalig aufgetreten sei, nachdem sie den Missbrauch als solchen verstehen konnten. Dabei kann davon ausgegangen werden, dass die Kognitionen bezüglich der Beschmutztheit sich im Laufe der Entwicklung der Betroffenen verändern, von diffusen Bewertungen im Kindesalter zu stark elaborierten Bewertungen im Erwachsenenalter.

Zum Verständnis der Ätiologie des Phänomens erscheinen darüber hinaus Befunde zu den Konzepten des soziomoralischen und interpersonellen Ekels bedeutsam: Soziomoralischer Ekel wird als eine Reaktion auf moralische Verletzungen definiert, die zeigen, dass eine Person moralisch verwerflich ist [Rozin et al., 2000], während interpersoneller Ekel durch den Kontakt mit unbekannten, kranken oder unmoralischen Menschen ausgelöst werden kann [Olatunji und Sawchuk, 2005]. Nach einer etwas vagen Definition von Rozin et al. [2000] dient interpersoneller Ekel dem Schutz der Seele und hält den persönlichen Bereich und die persönliche Integrität aufrecht. Er basiert unter anderem auf der Befürchtung, dass Charakteristika einer unmoralischen Person durch den
Kontakt zu dieser auf die eigene Person übergehen. Mehrere Studien zeigen, dass Beschreibungen sexualisierter Gewalt zu Ekelreaktionen gegenüber dem Täter führen [Jones und Fitness, 2008; Olatunji et al., 2008]. Nach dem von Rozin et al. [1986] postulierten Gesetz der Ansteckung («law of contagion») können Menschen zur Überzeugung gelangen, dass es durch einen kurzen Kontakt mit einer (in der Regel lebendigen) Quelle der Kontamination zu einer permanenten Übertragung von negativen Eigenschaften kommt. Vor diesem Hintergrund kann die mitunter mit dem Gefühl des Beschmutztseins einhergehende Überzeugung eingeordnet werden, dass ekelerregende Eigenschaften vom Täter durch Körperkontakt auf die Opfer übergegangen sind. Für die kognitive Verarbeitung bedeutsam könnten darüber hinaus gesellschaftliche Vorurteile gegenüber Opfern sexualisierter Gewalt sein. Es ist weiterhin davon auszugehen, dass durch Scham und Vermeidung dieses belastenden Erlebens eine Auseinandersetzung mit diesem und anderen Symptomen (z.B. durch die Kommunikation mit nahestehenden Personen) unterbleibt. Folglich können keine korrigierenden Informationen gesammelt werden, die die Überzeugung, beschmutzt, abstoßend oder wertlos zu sein, verändern.

Wertet man das Gefühl der Beschmutztheit im Sinne Rachmans [2004] als einen interpretativen Prozess (z.B. «Der Täter hat meinen Körper für immer befleckt.», «Teile von ihm sind auf mich übergegangen.»), scheint es naheliegend, die dysfunktionalen Kognitionen mithilfe geeigneter kognitiver Interventionen zu relativieren. Da darüber hinaus Ekel mit diesem Gefühl assoziiert zu sein scheint, könnte prolongierte Exposition zur Behandlung des Gefühls des Beschmutztseins aufgrund der Befunde zur langsamen oder fehlenden Habituation von Ekel [Mason und Richardson, 2010; Smits et al., 2002] und insbesondere soziomoralischem Ekel [Simpson et al., 2006] wenig erfolgversprechend sein. Die Entwicklung eines alternativen Ansatzes scheint indiziert.

\section{Vorschlag einer Intervention}

Zur Behandlung des Gefühls der Beschmutztheit nach sexuellem Missbrauch in der Kindheit schlagen wir zunächst eine klassische kognitive Umstrukturierung basierend auf aktiv erworbenem Wissen vor: Es wird die Überzeugung beschmutzt $\mathrm{zu}$ sein disputiert und mit Informationen zur Häufigkeit der Erneuerung der Oberhaut/Epidermis konfrontiert. Diese kognitive Intervention wird um eine imaginative Intervention ergänzt. Die Ergänzung scheint aus folgenden Gründen indiziert:

- Die Betroffenen geben oftmals an, dass sie rational wissen, dass es keine Spuren des Missbrauchs mehr an ihrem Körper gibt (= rationale Überzeugtheit). Dennoch fühle es sich so an (= emotionale Überzeugtheit) [Wells, 2000]. Wells [2000] als wichtiger Vertreter der Kognitiven Therapie postuliert, dass Imaginationen ein wichtiges Vehikel 
zur Modifikation des prozeduralen, impliziten Wissens darstellen. Nach Wells [2000] ist die emotionale Überzeugtheit im Bereich des impliziten Wissens einzuordnen.

- Strebt man eine Veränderung der emotionalen Überzeugtheit an, scheinen Ansätze besonders geeignet, die effizient Gefühle evozieren und verändern. Da die Prozessierung von Informationen während intensiver Emotionen mit einer deutlich gesteigerten perzeptuellen Verarbeitung einhergeht [z.B. Arntz et al., 2005], wird im Umkehrschluss davon ausgegangen, dass das Ziel einer Veränderung des Gefühls des Beschmutztseins über eine Veränderung der Wahrnehmungen (hier über Vorstellungsbilder) besonders günstig erreicht werden kann. Vorstellungsbilder/Imaginationen können dabei neben der visuellen auch andere Sinnesmodalitäten (z.B. kinästhetisch, olfaktorisch) umfassen [Kosslyn et al., 2001].

- Befunde der Grundlagenforschung weisen auf die Überlegenheit des Einsatzes von Imaginationen gegenüber verbalen Methoden in der Modifikation von Gefühlen hin [Holmes und Mathews, 2005; Holmes et al., 2008, 2009].

- Die Untersuchungsbefunde an Patientengruppen sprechen für den Einsatz imaginativer Verfahren (Bulimia nervosa: [Cooper et al., 2007]; Soziale Phobie: [Wild et al., 2007; Wheatley et al., 2007]). Auch in der Behandlung der PTBS [Arntz et al., 2007] hat sich der Einsatz von Imaginationen bewährt. In einem Übersichtsartikel veranschaulichen Holmes und Kollegen [2007] den Einsatz von Imagery Rescripting in der Kognitiven Verhaltenstherapie.

\section{Beschreibung der Cognitive Restructuring and Imagery Modification}

Da die Intervention bislang lediglich bei weiblichen Opfern sexualisierter Gewalt angewendet wurde, ist im Folgenden von Patientinnen die Rede. Wir gehen jedoch davon aus, dass auch männliche Betroffene von der Intervention profitieren.

Nach einer sorgfältigen Exploration der Qualität und Bedeutung des Gefühls des Beschmutztseins führt die Patientin - gegebenenfalls mit Unterstützung durch die Therapeutin eine etwa 10-minütige Recherche im Internet durch. Es soll die Frage beantwortet werden, wie häufig sich menschliche Epidermiszellen in verschiedenen Körperbereichen erneuern. Dies geschieht alle 4-6 Wochen, bei Schleimhäuten teils innerhalb weniger Tage [Braun-Falco et al., 2005].

Anschließend wird mithilfe eines Taschenrechners berechnet, wie häufig sich die Hautzellen seit dem Missbrauch im individuellen Fall in verschiedenen Bereichen des Körpers, die als besonders beschmutzt erlebt werden, erneuert haben. Die Bedeutung der Information für die Patientin wird hinterfragt. Danach wird der Patientin vermittelt, dass klinische Erfahrung zeigt, dass viele Betroffene sich durch die Information zur Hauterneuerung erleichtert fühlen und ihnen rational klar werde, dass sie heute nicht mehr beschmutzt sein können.
Allerdings würden viele Patientinnen weiterhin angeben, dass sie sich beschmutzt fühlen. Es wird erklärt, dass es typisch sei, dass das Gefühl dem Verstand «nachhinke» und es eines Hilfsmittels bedürfe, um die Information zum Gefühl zu bringen: das Vorstellungsbild. Die Patientin wird aufgefordert, selbst ein individuelles Bild zu generieren, das den Prozess des Abstreifens der alten Haut symbolisiert. Dieses Vorstellungsbild wird bezüglich seiner sensorischen Qualitäten detailliert ausgearbeitet, um so die Lebhaftigkeit der Imagination zu stärken [Kirn et al., 2009]. Es soll eine hinreichend große Anzahl von Propositionen (logische, nichtlinguistische Informationseinheiten im Langzeitgedächtnis) aktiviert werden, da dies für die Aktivierung eines emotionalen Netzwerks notwendig ist [Lang et al., 1983]. Es werden keinerlei Vorgaben bezüglich des Vorstellungsbildes gemacht, die Frauen werden aufgefordert, die Imagination so zu gestalten, wie es ihnen gefällt. Die Therapeutin instruiert lediglich, dass das Vorstellungsbild den Prozess der Hauterneuerung symbolisieren soll. Zunächst wird dann das Gefühl der Beschmutztheit für kurze Zeit in einem Ausmaß evoziert, wie es typischerweise im Alltag der Patientin auftritt. Anschließend wird in die Imagination zur Hauterneuerung übergeleitet. Dabei sollte deutlich werden, dass die Kontrolle auch während der Imagination bei der Patientin liegt. Die gesamte therapeutengeleitete Imagination ohne vorhergehende Erarbeitung umfasst durchschnittlich etwa $15 \mathrm{~min}$. Im Anschluss an die etwa 90-minütige Interventionssitzung erhalten die Patientinnen das individuelle Tonband (Kassettenaufzeichnung) zur Imagination des Gefühls der Beschmutztheit und der Hauterneuerung mit der Aufgabe, diese täglich anzuhören und die Imagination zu üben.

\section{Evaluation der Wirksamkeit der neuen Intervention}

Steil et al. [2011b] evaluierten die Wirksamkeit von Cognitive Restructuring and Imagery Modification (CRIM) im Rahmen einer 9 Patientinnen umfassenden Prä-Post-Pilotstudie. Vor der Behandlung (t0), danach (t1) und 6 Wochen nach Ende der Behandlung (t2) wurden Ratings bezüglich Intensität, Lebendigkeit und Unkontrollierbarkeit des Gefühls des Beschmutztseins erfasst, ferner wurden die damit verbundene Belastung und das Ausmaß der PTBS mithilfe der Posttraumatischen Diagnoseskala (PDS) [Griesel et al., 2006] erhoben. Beim Vergleich zwischen t0 und $\mathrm{t} 2$ zeigten sich große Effektstärken für die verschiedenen Aspekte des Gefühls des Beschmutztseins $(\mathrm{d}=1,83-2,79)$. Erstaunlicherweise zeigte sich auch für die Reduktion der gesamten PTBS-Symptomatik eine vergleichsweise große Wirksamkeit $(\mathrm{d}=0,99)$.

\section{Fallbeispiel}

Frau M., eine 43-jährige verheiratete Bürokauffrau, kommt mit einer PTBS infolge sexualisierter Gewalt im Alter von 9-11 Jahren, einer rezidivierenden depressiven Störung und einer Persönlichkeitsakzentuierung (4 


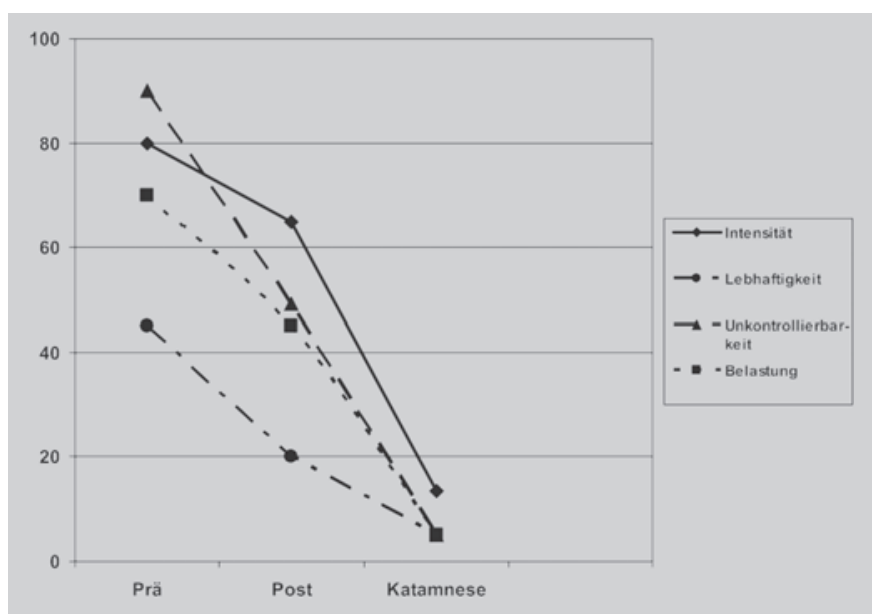

Abb. 1. Mittelwerte der Intensität, Lebhaftigkeit und Unkontrollierbarkeit des Gefühls des Beschmutztseins sowie der Belastung durch das Gefühl des Beschmutztseins zum Prä-, Post- und Katamnese-Messzeitpunkt zur Einzelfallstudie.

Kriterien der Borderline-Persönlichkeitsstörung) in Behandlung. In der Vorgeschichte beschreibt sie eine Anorexia nervosa. Die Diagnostik der genannten Störungen erfolgt mittels Strukturiertem Klinischen Interview für DSM-IV (Diagnostic and Statistical Manual of Mental Disorders IV) [Wittchen et al., 1997] und der Borderline-Sektion der International Personality Disorder Examination [Loranger et al., 1998]. Im Rahmen der vorliegenden Studie wurde ausschließlich das Gefühl des Beschmutztseins behandelt, während die Patientin auf einen Platz für eine Traumatherapie wartete. Die sexuellen Übergriffe seien von ihrem damaligen Schwimmlehrer ausgegangen, der mit dem Finger in sie eingedrungen sei und sie zur oralen Penetration gezwungen habe. Die PTBS-Symptomatik habe im Alter von 18 Jahren nach dem ersten Geschlechtsverkehr mit ihrem damaligen Freund begonnen. Das Gefühl des Beschmutztseins habe schon früher bestanden. In der Vorgeschichte haben eine stationäre Traumatherapie und 2 ambulante Behandlungen der Anorexia nervosa und PTBS stattgefunden. Frau M. gibt an, dass sie sich dreckig und besudelt fühle. Sie ekle sich vor ihrem Körper, insbesondere vor ihrem Genitalbereich. Oft denke sie, dass sie ihren Körper am liebsten loswerden würde. Die Anorexia nervosa in der Vorgeschichte sei aus der Intention entstanden, das Gefühl des Beschmutztseins zu beseitigen, indem sie nur noch Geist und möglichst wenig Körper sei. Frau M. gibt an, dass das Gefühl der Beschmutztheit permanent vorhanden sei. Zu einem Anstieg in der Intensität komme es durch Erinnerungen an den sexuellen Missbrauch und in sozialen Situationen mit körperlicher, aber auch zwischenmenschlicher Nähe. Typischerweise reagiere sie mit Rückzug und Ablenkungsversuchen. Darüber hinaus versuche sie, die Konfrontation mit ihrem Körper weitestgehend zu vermeiden. So gäbe es nur einen abgehängten Spiegel in ihrer Wohnung, sie trage stets sehr weite, dunkle Kleidung und vermeide Körperpflege, die über das notwendige Duschen hinausgehe.

Zur Überprüfung der Wirksamkeit der Intervention protokolliert Frau M. täglich am Ende des Tages die durchschnittliche Intensität, Lebhaftigkeit und Unkontrollierbarkeit des Gefühls des Beschmutztseins sowie die Belastung durch dasselbe auf visuellen Analogskalen (0-100). Im Vorfeld wird hierzu eine individuelle Ankersituation zur maximalen Ausprägung eingeführt. Die täglichen Protokollbögen werden von der Patientin in der Woche vor der Intervention ( $\mathrm{t} 1), 1$ Woche (t2) sowie 6 Wochen nach der Intervention (t3) ausgefüllt. Es wird ein Mittelwert für die gesamte Woche für die einzelnen Parameter berechnet. Weiterhin erhält die Patientin die PDS [Griesel et al., 2006] zur Erfassung der PTBS-Symptomatik zu t1, t2 und t3. Die PDS ist ein Selbstbeurteilungsinstrument, das als Screening-Instrument nach DSM-IV geeignet ist und den Schweregrad der Symptomatik erfasst. Scores zwischen 10 und 20 weisen auf eine leichte bis mittlere, Scores zwischen 20 und 30 auf eine mittelschwere, Scores über 30 auf eine schwere PTBS-Symptomatik hin.

In der Behandlungssitzung wird nach sorgfältiger Exploration der Merkmale des Gefühls des Beschmutztseins (z.B. klebrig, feucht, juckend, permanent) die Patientin zur Internetrecherche zur Hauterneuerung angeleitet. Sie berechnet, dass sich sämtliche vom Missbrauch betroffenen Hautzellen seit dem letzten Übergriff mindestens 372-mal erneuert haben. Dies bedeute, dass sie dem Täter heute überlegen sei, da er keinen Einfluss mehr auf sie und ihren Körper ausüben könne. Im Anschluss wird ein individuelles Vorstellungsbild erarbeitet: Frau M. generiert die Vorstellung, von einem warmen, trockenen Wind an einem Strand «umspült» zu werden, der dafür sorgt, dass die klebrigen, grauen, feuchten, juckenden Hautpartikel getrocknet und schließlich abgelöst werden, sodass letztendlich neben ihr ein Berg der alten, vertrockneten Hautzellen liegt. Darunter komme allmählich frische, weiche, reine Haut hervor, die angenehm duftet. Frau M. spürt die Sonne und den Wind auf ihrer neuen Haut. Sie lässt den Berg alter Hautzellen zurück im Bewusstsein, dass ihre heutige Haut und ihr heutiger Körper nichts mehr mit dieser alten Haut zu tun haben, die vom Täter berührt wurde. Nachdem das individuelle Vorstellungsbild zur Zufriedenheit der Patientin erarbeitet wurde, wird die Patientin instruiert, sich an eine kürzlich stattgefundene Begebenheit zu erinnern, die mit dem Gefühl des Beschmutztseins einherging. Die Patientin wählt eine Szene der letzten Woche, in der sie sich nach dem Duschen abtrocknete und ihr Mann im Bad erschien, was in ihr den Gedanken auslöste, dass er sich vor ihr ekeln müsse. Durch die Imagination dieser Szene gelingt es Frau M. rasch, das Gefühl des Beschmutztseins zu aktivieren. Von außen zu beobachten ist, dass sie zeitgleich eine typische Schamhaltung (gesenkter Kopf, Zusammensacken, Krümmung der Körperachse) einnimmt. Die Therapeutin leitet bei einer Intensität des Gefühls des Beschmutztseins von 70 in die Vorstellung zur Hauterneuerung über und empfiehlt der Patientin dabei das Einnehmen einer schamkonträren Haltung (aufrechte, gerade Körperhaltung, leichtes Lächeln). Die Patientin kommt der Empfehlung nach und verändert ihre Körperhaltung. In der Nachbesprechung der Imaginationsübung gibt Frau M. an, dass sie erstaunt gewesen sei, wie schnell sich durch die Übung das Gefühl der Beschmutztheit verringert habe. Bis zum abschließenden Treffen zur Nachbesprechung nach einer Woche (à $50 \mathrm{~min}$ ) hört die Patientin das Tonband mit der Imagination täglich. Der Verlauf der Ratings zeigt eine mäßige Veränderung in der Intensität des Gefühls des Beschmutztseins, während Lebhaftigkeit, Unkontrollierbarkeit und Belastung deutlich reduziert sind. Die Patientin gibt an, nicht nur regelmäßig mithilfe der Kassette zu üben, sondern auch im Alltag ihr Vorstellungsbild dem Gefühl von Beschmutztheit entgegenzusetzen. 6 Wochen nach der Intervention zeigt sich in den zugesendeten Daten ein deutlicher Abfall im Gefühl des Beschmutztseins (Abb. 1). Auch in der PDS zeigt sich eine Verbesserung der Symptomatik (Summenwerte: $\mathrm{t} 1=17, \mathrm{t} 2=13, \mathrm{t} 3=9$ ). Diese ist insbesondere auf eine Reduktion innerhalb der Subskala «Wiedererleben» zurückzuführen.

In einem Telefonat nach 4 Monaten berichtet Frau M., dass es ihr nun erstmalig seit langer Zeit wieder möglich sei, Nähe zu ihrem Partner zuzulassen, und dass sie ihren Körper seit Jahren zum ersten Mal wieder eingecremt habe.

\section{Diskussion}

Das Gefühl des Beschmutztseins stellt ein Phänomen bei Opfern sexualisierter Gewalt dar, das bislang in der einschlägigen Literatur wenig beschrieben wurde und für das kein Behandlungsansatz existierte. Im vorliegenden Artikel wird auf dessen Manifestation und resultierende Konsequenzen sowie mögliche theoretische Hintergründe zur Pathogenese eingegangen. Ein neu entwickeltes Behandlungskonzept basierend 
auf kognitiver Umstrukturierung und Imagination wird vorgestellt. Die Falldarstellung veranschaulicht die Behandlungsmethode und zeigt, dass durch eine lediglich eine Sitzung umfassende Intervention eine deutliche Reduktion im Gefühl des Beschmutztseins erreicht werden konnte. Dies ist umso bemerkenswerter als die Patientin seit Jahren massiv unter der Symptomatik gelitten und ihr Leben darauf eingerichtet hatte. Entscheidend für die rasche Verbesserung scheinen das Erleben von Kontrolle im Umgang mit dem Gefühl und der resultierende Anstieg in der erlebten Selbstwirksamkeit zu sein. Als weitere Wirkmechanismen können die Modifikation der rationalen Überzeugtheit beschmutzt zu sein infolge der Internetrecherche und Disputation, die Schamreduktion durch Mitteilen des Gefühls des Beschmutztseins gegenüber der Therapeutin, Entpathologisierung durch Information, dass andere Opfer sexualisierter Gewalt ebenfalls unter einem Gefühl des Beschmutztseins leiden, sowie die angstreduzierende Konkretisierung der Eigenschaften des diffusen Gefühls des Beschmutztseins diskutiert werden. Bislang unklar ist, in welchem Ausmaß die Wirksamkeit der Imagination auf dem Expositionselement (Evozieren des Gefühls des Beschmutztseins) bzw. die kognitive Umstrukturierung und die Veränderung der Imagination (Vorstellung zur Hauterneuerung) beruht. Dies ist in zukünftigen Dismantling-Studien zu klären.

Bemerkenswert sind die ausgeprägte weitere Verbesserung der Gefühls des Beschmutztseins in der Katamnese und ein deutlicher Abfall in der PTBS-Symptomatik.

Erste Ergebnisse einer unkontrollierten Pilotstudie an 9 Patientinnen [Steil et al., 2011b] zur Wirksamkeit der beschriebenen Intervention legen nahe, dass mit ihr das Gefühl der Beschmutztheit und die Symptomatik der PTBS reduziert werden können. Die Ergebnisse der derzeit durchgeführten randomisierten und kontrollierten Studie stehen jedoch noch aus. Kritisch bewertet werden muss, dass bislang keine Daten zur längerfristigen Wirksamkeit der Methode über die 6-wöchige Katamnese hinaus existieren.

\section{Integration in einen Behandlungsplan, Indikation und Kontraindikation}

In der beschriebenen Fallstudie wurde ausschließlich das Gefühl des Beschmutztseins behandelt. Im klinischen Alltag sollte die Behandlungsmethode in einen traumazentrierten Gesamtbehandlungsplan integriert werden. Ursprünglich wurde sie im Rahmen der Dialektisch Behavioralen Therapie für PTBS nach sexualisierter Gewalt in der Kindheit und Jugend [DBT-PTSD; Steil et al., 2011a] entwickelt, kann jedoch mit jedem anderen traumafokussierten Verfahren [z.B. Ehlers et al., 2005; Resick et al., 2008] kombiniert werden. Der behandelnde Therapeut sollte dabei über Training und Erfahrung in kognitiver Therapie verfügen. Günstig erscheint ein Einsatz der beschriebenen Methode bei ausgeprägtem Gefühl der Beschmutztheit im Vorfeld der Exposition, da die zu erwartende rasche Wirksamkeit der Methode die Motivation zur Exposition steigern dürfte. Weiterhin ist eine Anwendung der Intervention bei ausbleibender Remission des Gefühls des Beschmutztseins nach ansonsten erfolgreicher Exposition indiziert. Als Kontraindikation kann das Vorliegen einer akuten psychotischen Symptomatik [Kossak, 2004] gewertet werden. Bei geringer Imaginationsfähigkeit scheint ein vorhergehendes Imaginationstraining indiziert.

Zusammengefasst weisen die vorliegende Einzelfallstudie sowie erste Pilotdaten auf eine gute Wirksamkeit und hohe Effizienz der Kurzintervention zur spezifischen Behandlung des Gefühls des Beschmutztseins nach sexualisierter Gewalt in der Kindheit und Jugend hin.

\section{Disclosure Statement}

Es bestehen bei keinem der Autoren Interessenskonflikte.

\section{Literatur}

Arntz A, DeGroot C, Kindt M: Emotional memory is perceptual. J Behav Ther Exp Psychiatry 2005;36 19-34.

Arntz A, Tiesema M, Kindt M: Treatment of PTSD: A

comparison of imaginal exposure with and without imagery rescripting. J Behav Ther Exp Psychiatry 2007:38:345-370.

Braun-Falco O, Plewig G, Wolff $\mathrm{HH}$, et al.: Dermatologie und Venerologie. Berlin, Springer, 2005

Cooper M, Todd G, Turner H: The effects of using im-

agery to modify core emotional beliefs in bulimia nervosa: An experimental pilot study. J Cogn Psychother 2007;21:117-121.

DeSilva P, Marks M: The role of traumatic experi-

ences in the genesis of obsessive-compulsive disorder. Behav Res Ther 1999;37:941-951.

Ehlers A, Clark DM: A cognitive model of posttraumatic stress disorder. Behav Res Ther 2000;38:319345 .
Ehlers A, Clark DM, Hackmann A, et al.: Cognitive therapy for posttraumatic stress disorder: Development and evaluation. Behav Res Ther 2005;43:413431

Fairbrother N: An investigation of the Ehlers-Clark cognitive theory of PTSD and the phenomenon of mental pollution. Doctoral dissertation, University of British Columbia, Vancouver, 2002.

Fairbrother N, Rachman S: Feelings of mental pollu-

tion subsequent to sexual assault. Behav Res Ther 2004;42:173-189.

Gershuny BS, Baer L, Jenike MA, et al.: Comorbid

posttraumatic stress disorder: Impact on treatment outcome for obsessive-compulsive disorder. Am J Psychiatry 2002;159:852-854.

Griesel D, Wessa M, Flor H: Psychometric properties

of the German version of the Posttraumatic Diagnostic Scale (PDS). Psychol Assess 2006;18:262268.
Hathaway LM, Boals A, Banks JB: PTSD symptoms and dominant emotional response to a traumatic event: An examination of DSM-IV Criterion A2. Anxiety Stress Coping 2010;23:119-126.

Herman JL: Complex PTSD: A syndrome in survivors of prolonged and repeated trauma. J Trauma Stress 1992;5:377-391.

Holmes EA, Arntz A, Smucker MR: Imagery rescript-

ing in cognitive behaviour therapy: Images, treatment techniques and outcomes. J Behav Ther Exp Psychiatry 2007;38:297-305.

Holmes EA, Lang TJ, Shah DM: Developing interpretation bias modification as a 'cognitive vaccine' for depressed mood: Imagining positive events makes you feel better than thinking about them verbally. J Abnorm Psychol 2009;118:76-88.

Holmes EA, Mathews A: Mental imagery and emo-

tion: A special relationship? Emotion 2005;5:489 497. 
Holmes EA, Mathews A, Mackintosh B, et al.: The causal effect of mental imagery on emotion assessed using picture-word cues. Emotion 2008;8 395-409.

Jones A, Fitness J: Moral hypervigilance: The influ-

- ence of disgust sensitivity in the moral domain. Emotion 2008;8:613-627.

Kirn T, Echelmeyer L, Engberding M: Imagination in der Verhaltenstherapie. Heidelberg, Springer, 2009.

Kossak HC: Hypnose: Lehrbuch für Psychotherapeuten und Ärzte, ed 4. Weinheim, Beltz, 2004.

Kosslyn SM, Ganis G, Thompson WL: Neural foundations of imagery. Nat Rev Neuro Sci 2001;2:635642.

Lang PJ, Levin DN, Miller GA, et al.: Fear behaviour,

fear imagery, and the psychophysiology of emotion The problem of affective response integration. J Abnorm Psychol 1983;92:276-306.

Loranger AW, Sartorius N, Andreoli A, et al.: Deutschsprachige Fassung der International Personality Disorder Examination: IPDE. Genf, WHO, 1998.

Mason EC, Richardson R: Looking beyond fear: The

extinction of other emotions implicated in anxiety disorders. J Anxiety Disord 2010;24:63-70.

Olatunji BO, Haidt J, McKay D, et al.: Core, anima

reminder, and contamination disgust: Three kinds of disgust with distinct personality, behavioral, physiological, and clinical correlates. J Res Pers 2008;42:1243-1259.
Olatunji BO, Sawchuk CN: Disgust: Characteristic

features, social manifestations, and clinical implications. J Soc Clin Psychol 2005;24:932-962.

Power M, Dalgleish T: Cognition and Emotion: From Order to Disorder. New York, Psychology Press, 2008.

Rachman S: Case histories and shorter communica-

tions: Pollution of the mind. Behav Res Ther 1994; 32:311-314.

Rachman S: Fear of contamination. Behav Res Ther 2004;42:1227-1255.

Rachman S: Fear of Contamination: Assessment and Treatment. Oxford, Oxford University Press, 2006.

Resick PA, Galovski TE, O'Brien Uhlmansick M, et

al.: A randomized clinical trial to dismantle components of cognitive processing therapy for posttraumatic stress disorder in female victims of interpersonal violence. J Consult Clin Psychol 2008;76:243-258.

Rozin P, Haidt J, McCauley CR: Disgust; in Lewis M, Haviland JM (eds): Handbook of Emotions. New York, Guilford Publications, 2000, pp 637653.

Rozin P, Millman L, Nemeroff C: Operation of the

laws of sympathetic magic in disgust and other domains. J Pers Soc Psychol 1986;50:703-712.

Simpson J, Carter S, Anthony SH, et al.: Is disgust a

homogenous emotion? Motiv Emot 2006:30:3141.
Smits J, Telch M, Randall P: An examination of the

decline in fear and disgust during exposure-based treatment. Behav Res Ther 2002;40:1243-1253.

Steil R, Dyer A, Priebe K, et al.: Dialectical behavior

therapy for posttraumatic stress disorder related to childhood sexual abuse: A pilot study of an intensive residential treatment program. J Trauma Stress 2011a;24:102-106.

Steil R, Jung K, Stangier U: Efficacy of a two-session

$\checkmark$ program of cognitive restructuring and imagery modification to reduce the feeling of being contaminated in adult survivors of childhood sexual abuse: A pilot study. J Behav Ther Exp Psychiatry 2011b; 42:325-329.

Wells A: Emotional Disorders and Metacognition: Innovative Cognitive Therapy. Chichester, Wiley, 2000.

Wheatley J, Brewin CR, Patel T, et al.: „I'll believe it

when I can see it": Imagery rescripting of intrusive sensory memories in depression. J Behav Ther Exp Psychiatry 2007;38:371-385.

Wild J, Hackman A, Clark DM: When the present vis-

its the past: Updating traumatic memories in social phobia. J Behav Ther Exp Psychiatry 2007;38:386401.

Wittchen H-U, Zaudig M, Fydrich T: Strukturiertes Klinisches Interview für DSM-IV (SKID). Göttingen, Beltz-Test, 1997. 